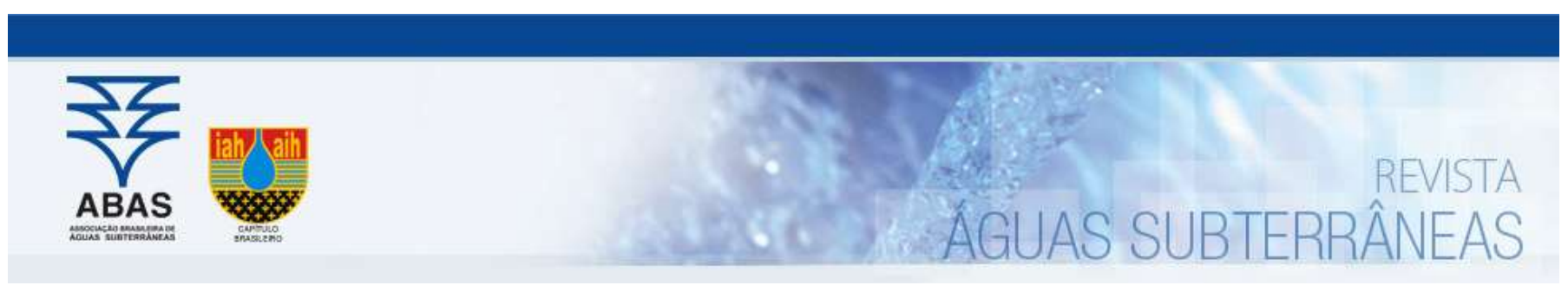

Artigos

\title{
Aplicação do método de fluorescência induzida por laser na caracterização de áreas contaminadas com resíduos oleosos
}

\section{Application of laser-induced fluorescence method in oil waste contaminated site}

\author{
Fernando de Melo Krahenbuhl1; Elias Hideo Teramoto2; Chang Hung Kiang ${ }^{3} \bowtie$ \\ 1 Petrobras - Petróleo Brasileiro SA. \\ 2 Centro de Estudos Ambientais (UNESP), Campus de Rio Claro, São Paulo, Brasil \\ 3 Departamento de Geologia Aplicada (UNESP), Campus de Rio Claro / Lebac - Laboratório de Estudo de Bacias (UNESP), Campus de \\ Rio Claro, São Paulo, Brasil
}

$\triangle$ fernandokrahenbuhl@hotmail.com, teramoto@rc.unesp.br, chang@rc.unesp.br

\begin{tabular}{ll} 
& Resumo \\
\cline { 2 - 2 } Palavras-chave: & A aplicação de perfilagem contínua, por meio do método de fluorescência induzida por laser (LIF), em uma área urbana com \\
Fluorescência Induzida por Laser. & disposição pretérita de resíduos oleosos (superior a 30 anos), mostrou-se útil na delimitação tridimensional de resíduos oleosos \\
Investigação Ambiental em Alta & em subsuperfície, indicando em tempo real e com baixo investimento a área passível de remediação. A comparação com dados \\
Resolução. & obtidos por outras formas de investigação ambiental indica que o LIF é eficiente na detecção em campo, tanto de resíduos oleosos \\
Resíduo Oleoso. & como de material oleoso impregnado no solo, independente do seu grau de intemperismo e da heterogeneidade na distribuição \\
Hidrocarboneto Poliaromático. & espacial. As respostas de fluorescência foram coerentes com o tipo de material encontrado, em que predominam hidrocarbonetos \\
& aromáticos policíclicos de cadeias maiores. O método foi sensível mesmo no caso de lentes delgadas de resíduos.
\end{tabular}

Abstract

Keywords:

Laser Induced Fluorescence. High-Resolution Site Characterization. Oil Waste.

Poliaromatic Hydrocarbon.

Revisado por pares.

Recebido em: 24/08/2017

Aprovado em: 08/02/2018

\begin{abstract}
The application of the Laser Induced Fluorescence (LIF) method in an urban area with oil waste deposited over 30 years shown good results and has proven to be an important tool to be used in tridimensional delimitation of oil residue plumes, specifying in real time and at low cost the region where remediation should be undertaken. Comparing the results obtained by other environmental investigation methods LIF has demonstrated to be efficient in field detection of oil residues as well as oil impregnated soil, independent of the degree of weathering and heterogeneity of spatial distribution. The response to fluorescence was coherent with the type of material that predominates composed of long-chain-hydrocarbons. The method was sensible to detect even thin lenses of residues.
\end{abstract}

DOI: http://dx.doi.org/10.14295/ras.v32i1.28923

\section{INTRODUÇÃO}

Nas últimas décadas, novas tecnologias de investigação e monitoramento de áreas contaminadas têm sido desenvolvidas, principalmente objetivando melhora na eficiência, aceleração dos resultados e racionalização de gastos (BUJEWSKI; RUTHERFORD, 1997; MONDELLI et al., 2014; ITRC, 2015). Nos Estados Unidos da América (EUA) são atualmente adotadas duas abordagens em investigações ambientais (RIYIS, 2012): a "Abordagem Tríade" (TRIAD Approach) e a "Avaliação Expedita da Área" (Expedite Site Assessment - ESA). Nos dois casos, o objetivo principal é realizar investigações ambientais com alta qualidade e densidade de informações, no menor tempo e custo possíveis, fazendo uso de recursos tecnológicos avançados.

A aplicação da Abordagem Tríade permite a elaboração de um modelo conceitual mais apurado, capaz de distinguir caracteres ticas particulares das contaminações, para as quais as decisões sobre os métodos de remediação a serem aplicados são diferentes (CRUMBLING, 2004). Ainda conforme Crumbling (2004), a Abordagem Tríade representa um avanço no gerenciamento de áreas contaminadas. A abordagem é baseada em três elementos, considerados como seus pontos fundamentais, que são o Planejamento Sistemático do Projeto, as Estratégias Dinâmicas de Trabalho e o uso de Sistema de Aquisição de Dados em Tempo Real.

A abordagem Tríade se apoia nas ferramentas de investigação ambiental de áreas contaminadas em alta resolução (High-ResoIution Site Characterization). Segundo EPA (2013), HRSC representam a aplicação de estratégias e técnicas usadas em escala e densidade de dados apropriados para definir a distribuição dos contaminantes e o contexto físico no qual estão inseridos, com grande precisão, subsidiando a recuperação da área de forma 
mais rápida e eficaz. Na aplicação da HRSC são utilizadas ferramentas e técnicas de investigação in situ, comumente por meio da penetração de sensores no meio investigado, que fornecem dados físicos e/ou químicos dos compartimentos e da contaminação em tempo real. Essas ferramentas incluem dispositivos de amostragem de gás do solo (ativo ou passivo), perfilagem hidroestratigráficas do solo, verificação da qualidade de água subterrânea, detecção direta de contaminantes, medidores de vazão, sistemas contínuos de amostragem e ferramentas de amostragem específicas do meio (USEPA, 2013). São geradas grandes quantidades de informações em detalhe, permitindo a caracterização vertical e horizontal da contaminação, com dados de distribuição, volume e massa, de modo a reduzir as incertezas e aprimorar o modelo conceitual (RIYIS, 2012; HADLEY; NEWELL, 2014; ITRC, 2015; SANTOS et al., 2015). Dados gerados por essas técnicas têm largas aplicações, inclusive em perícias legais de casos judiciais (MURPHY e MORRISON, 2014).

Conforme Riyis (2012), as técnicas de alta resolução podem ser divididas em dois grupos principais, conforme o objetivo: caracterização química e caracterização física. A tecnologia LIF tem sido usada como ferramenta auxiliar na investigação de contaminações em fase livre e residual de hidrocarbonetos em subsuperfície (USEPA, 2011; LAMB, 2012; ITRC, 2015). No Brasil ainda são incipientes os estudos baseados nessa tecnologia. Conforme Isler et al. (2013), a aplicação experimental de LIF em área contaminada, com presença de fase livre e residual de querosene de aviação, apresentou resultados condizentes para o produto analisado

Buscando atender às demandas do gerenciamento ambiental, várias empresas desenvolveram tecnologias de investigação baseadas em princípios ópticos que podem ser aplicados diretamente em campo. Algumas dessas tecnologias foram incorporadas a equipamentos como o Ultra Violet Optical Screening Tool (UVOST), desenvolvido pela Dakota Technologies, que usa a Fluorescência Induzida por Laser (LIF) para identificação in situ de contaminações por determinados tipos de hidrocarbonetos.

A técnica LIF é aqui utilizada em uma área contaminada no município de São Sebastião (Figura 1), com vistas a verificar sua aplicabilidade na identificação e delimitação de resíduos oleosos antigos, diretamente em campo. A comparação dos resultados com dados gerados por métodos tradicionais de diagnóstico ambiental permitiu a correlação e o melhor entendimento das variáveis envolvidas no processo de caracterização acelerada.

\section{MATERIAIS E MÉTODOS}

\subsection{Descrição da área contaminada e ensaios realizados}

Estudos de diagnóstico geoambiental indicam que se tratava de antiga várzea, formada pelo represamento de drenagem local em zona rural. A área foi aterrada ainda na década de 1980, com materiais diversos, sendo posteriormente alvo de ocupação urbana. Foram depositados no local resíduos oleosos, lixo urbano, entulho de construção civil, areia de praia etc. (GEORADAR, 2006, 2007a, 2007b; GEOKLOCK, 2011; FUNDUNESP, 2011). Além disso, análises químicas acusaram presença de coliformes fecais no solo e na água subterrânea, provavelmente oriundos de fossas sépticas ou de vazamentos na rede de esgoto local (FUNDUNESP, 2013).

$\mathrm{Na}$ área de várzea, onde predominavam condições anaeróbicas, ocorrem localmente lentes de argila orgânica de coloração escura (cinza a cinza esverdeada), que em sondagens convencionais se mostram visualmente muito semelhantes aos resíduos oleosos. Este fato dificultou o mapeamento das ocorrências de material oleoso (borras e solo impregnado) in situ, conforme GEORADAR (2006, 2007a, 2007b) e GEOKLOCK (2011). Em razão da forma de disposição do material - não segregado e misturado ao solo local -, as ocorrências de material oleoso são muito heterogêneas, ocorrendo na forma de lentes descontínuas, de espessuras centimétricas a métricas, com extensão lateral e profundidades muito variáveis (GEOKLOCK, 2011) (Figura 1).

Por serem depósitos antigos, com idade estimada superior a 30 anos, os resíduos dispostos no aterramento, inclusive os oleosos, encontram-se em adiantado processo de intemperização (degradação) (GEORADAR, 2006; 2007a e 2007b). Em locais onde houve coleta de amostras com profundidades distintas, as amostras mais superficiais apresentaram estágios mais avançados de degradação que as amostras mais profundas, indicando uma tendência de maior degradação em direção à superfície do solo.

Figura 1 - Seção geológica SW - NE da área (FUNDUNESP, 2011)

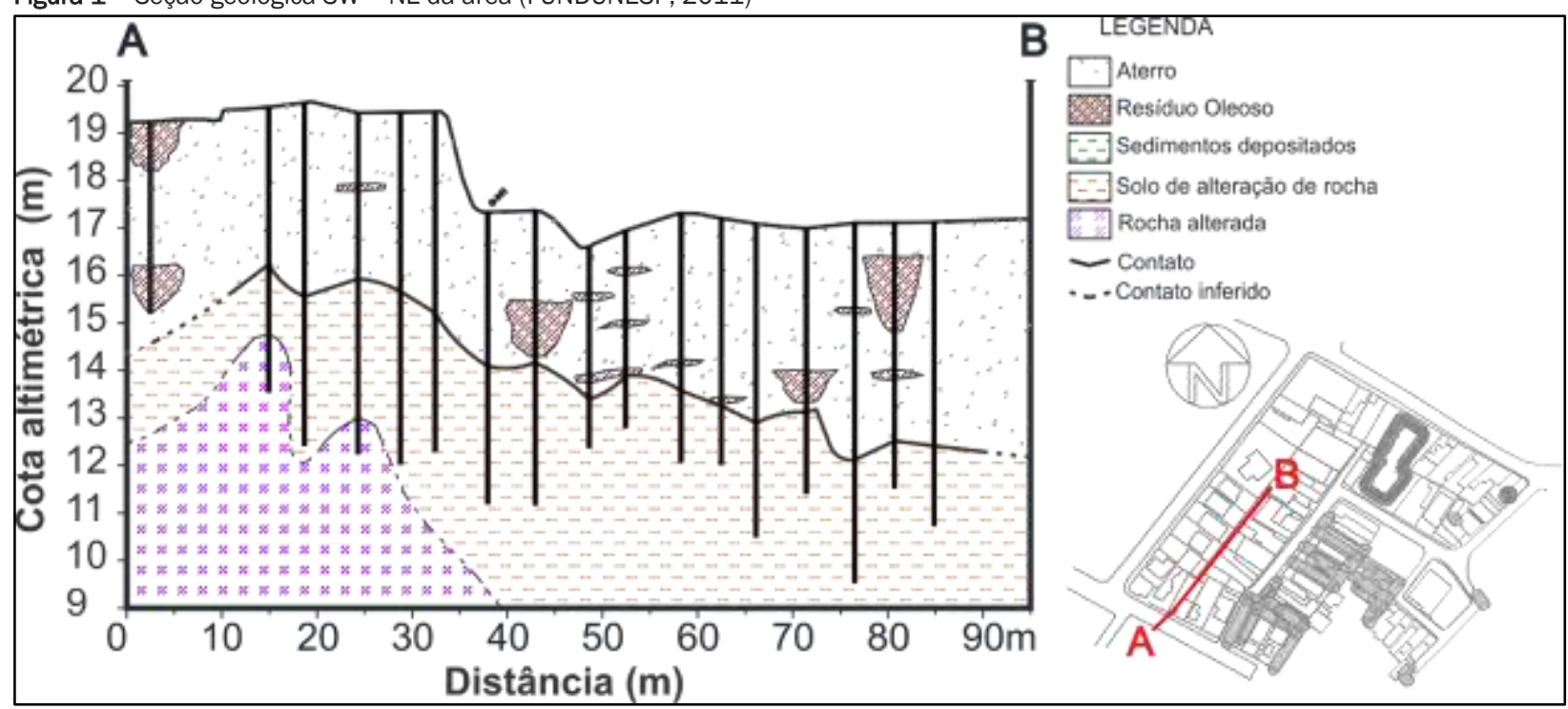


Nas análises químicas de solo e água subterrânea poucos foram os compostos orgânicos identificados acima dos limites de quantificação dos métodos aplicados (GEOKLOCK, 2011). Este fato tem sido atribuído à degradação dos resíduos em decorrência do intemperismo atuante ao longo do tempo. Apenas localmente, Hidrocarbonetos Totais de Petróleo (TPH) no solo e benzo(b)fluoranteno no solo e na água subterrânea foram identificados acima dos valores orientadores adotados (GEOKLOCK, 2011). Segundo a norma ABNT/NBR 10.004/2004, os resíduos são enquadrados na Classe II A (Não Inerte), pela presença de metais acima do Valor Máximo Permitido (VMP). Mesmo na análise direta do resíduo oleoso, compostos orgânicos não foram identificados (GEOKLOCK, 2011).

De modo a favorecer a correlação dos resultados, as perfilagens do presente estudo foram realizadas próximo aos locais onde foram executadas sondagens convencionais em trabalhos anteriores (Figura 2).

Figura 2 - Localização das perfilagens (LIF) e sondagens

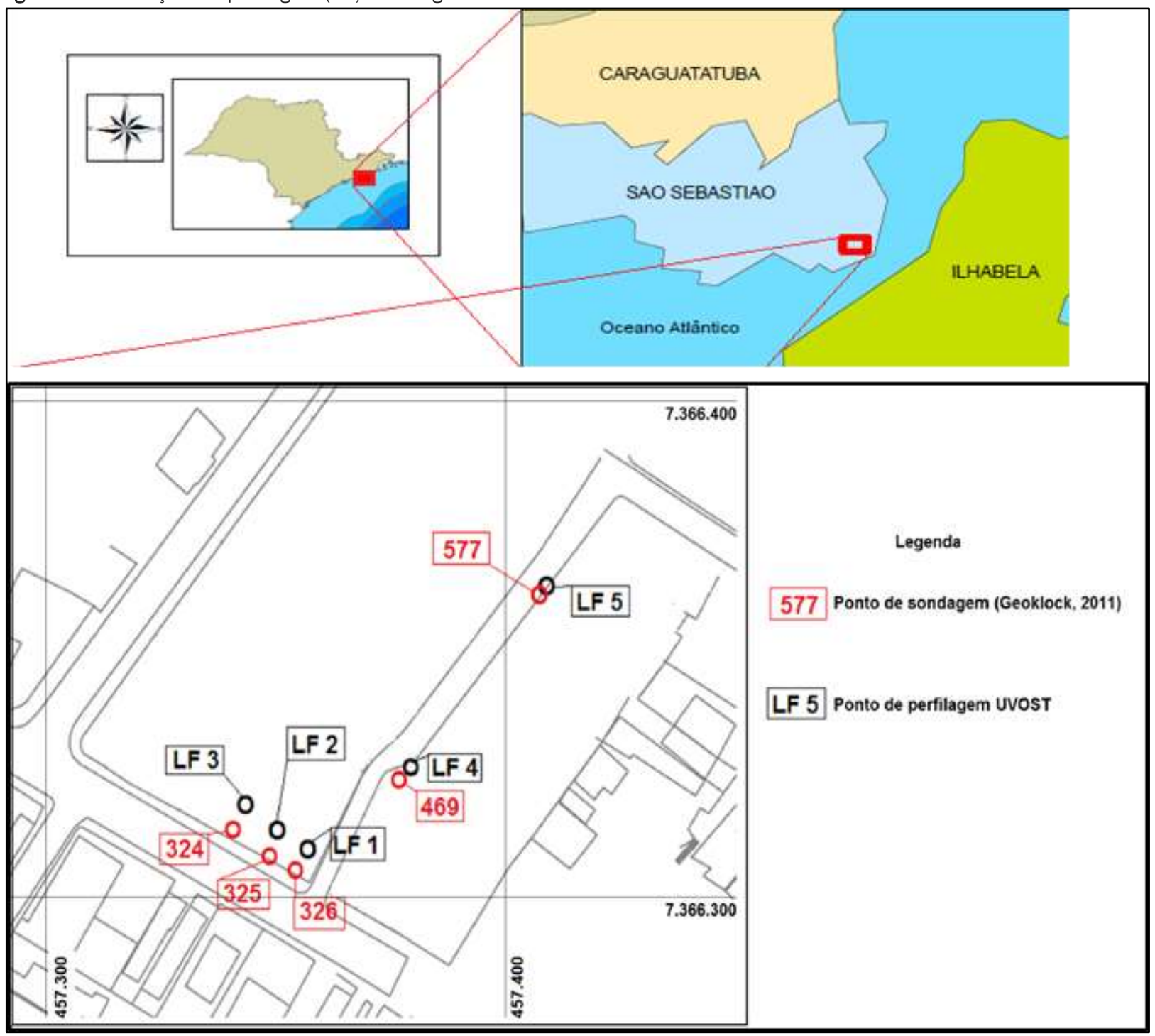

\subsection{Florescência induzida por laser}

A técnica desenvolvida pela Dakota Technologies no UVOST se baseia na propriedade de alguns Hidrocarbonetos Policíclicos Aromáticos (HPA) em liberar fótons de comprimentos de onda maiores, quando estimulados pela luz, graças às características dos anéis aromáticos de sua estrutura (HESS; HAMILTON, 1995; KARLITSCHEK et al., 1998). No caso específico do LIF, é captada a fluorescência emitida pelos HPA quando expostos à radiação ultravioleta. Como o espectro de emissão é único para cada HPA, não alterando em função do comprimento da onda de excitação, é possível obter dados qualitativos.
Segundo Bravo (2005), os HPA possuem propriedades próximas às ideais para identificação por fluorescência. Esses compostos possuem grande capacidade de absorção de energia, sendo que o máximo dessa absorção ocorre próximo do espectro da luz ultravioleta e da luz visível. Adicionalmente, a fluorescência desses compostos persiste por algum tempo, na faixa de dezenas a centenas de nanosegundos, facilitando sua detecção. A grande sensibilidade para emissão de fluorescência resultou na diminuição dos limites de detecção para a maioria dos HPA. Outra característica é que a intensidade da fluorescência apresenta relação direta (linear) com a concentração até aproximadamente seis ordens de magnitude. 
Na perfilagem LIF, utilizando a ferramenta UVOST (Figura 3) fabricado pela empresa Dakota Technologies com capacidade para efetuar ensaios de fluorescência (LIF), cor do solo e condutividade elétrica (CE). 0 equipamento foi configurado de forma que fosse possível realizar ensaios simultâneos de UVOST e CE ou SCOST e CE, mas não UVOST e SCOST. Dessa forma, após a execução do UVOST a sonda era deslocada lateralmente alguns centímetros para a realização do SCOST usando os mesmos equipamentos.
No ensaio de LIF um emissor fornece luz ultravioleta na faixa de $308 \mathrm{~nm}$ que é transmitida por cabos óticos internos ao sistema de cravação (Figura 4) até que incida no solo através de uma janela de safira (Figura 5). Em presença de hidrocarbonetos ocorre a fluorescência, que é captada pela mesma janela; o sinal é transmitido para a superfície (Figura 5), onde é convertido e o resultado apresentado na forma de gráficos com quatro curvas espectrais (callouts) (Figura 6).

Figura 3 - Aparelho UVOST da Dakota Technologies para as técnicas LIF, CE e SCOST

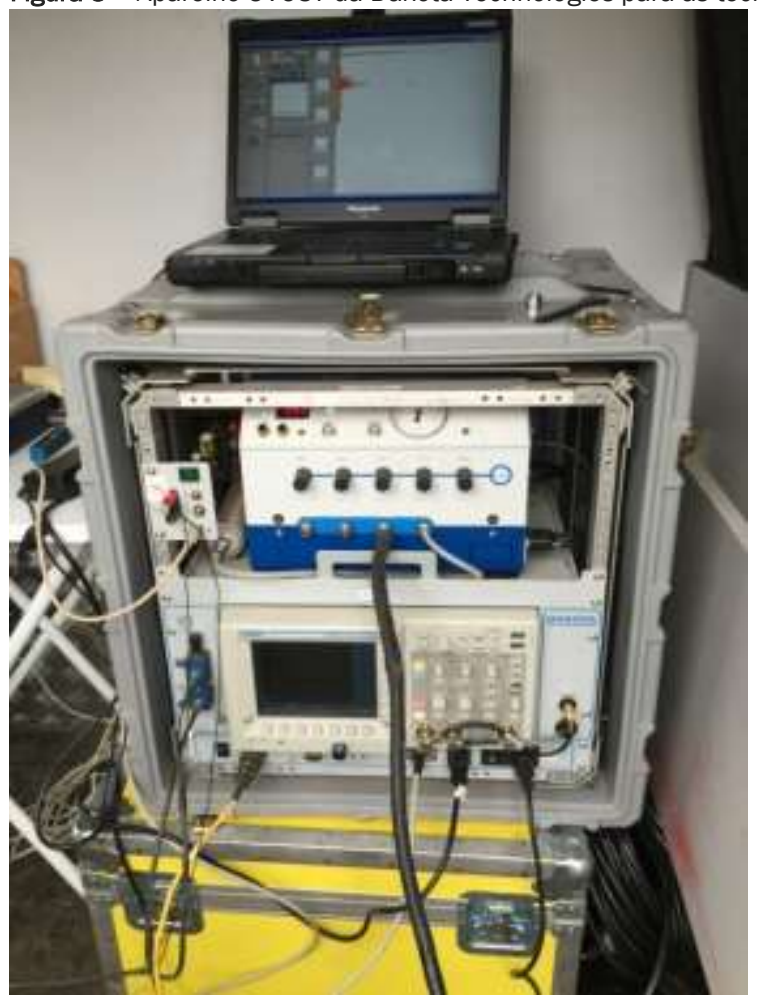

Figura 4 - Cravação da ponteira por Geoprobe

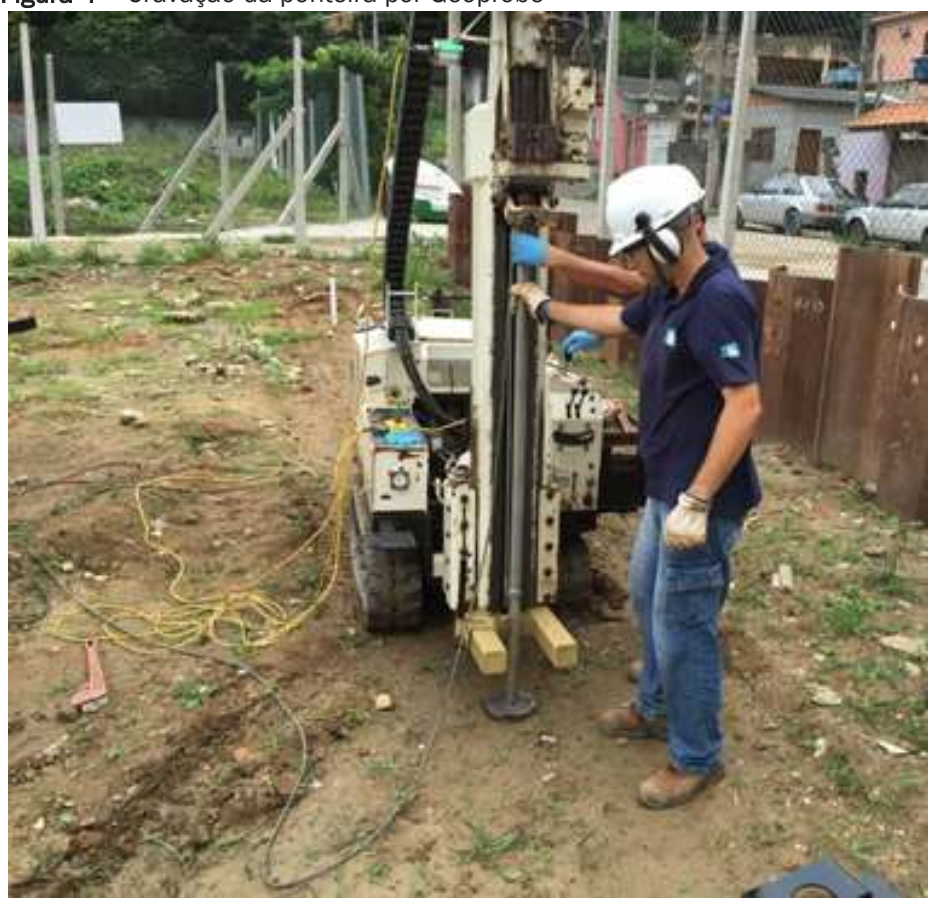


Figura 5 - Esquema da ponteira de cravação com indicação dos raios UV, a fluorescência, da janela de safira (LIF e SCOST) e do dipolo elétrico $(\mathrm{CE})$

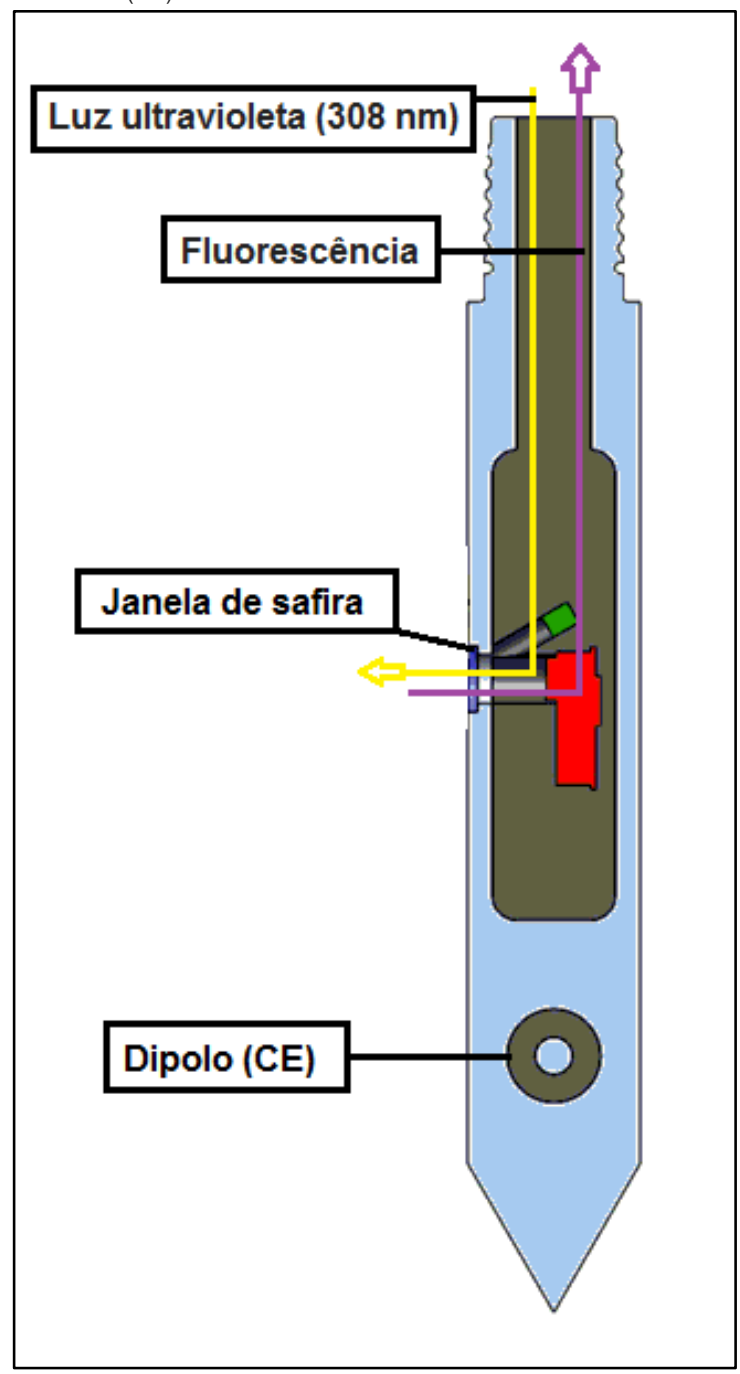

A emissão de fluorescência varia de forma direta com número de anéis aromáticos do HPA (tamanho) e o grau de substituição. Em geral, HPA com massas moleculares maiores apresentam absorbância e emissão de comprimentos de ondas maiores. 0 sensor capta intervalos de comprimentos de onda específicos que são característicos dos compostos: $350 \pm 20 \mathrm{~nm}$ para querosene de aviação e gasolina; $400 \pm 20 \mathrm{~nm}$ para diesel; $450 \pm 20 \mathrm{~nm}$ para creosoto; e $500 \pm 20 \mathrm{~nm}$ para hidrocarbonetos não especificados (Figura 6).

Com base na comparação do percentual de resposta em relação ao sinal emitido (Reference Emitter - \%RE), com um padrão do fabricante do equipamento (waveform), é possível obter-se uma análise qualitativa. A cada faixa citada acima é atribuída uma cor diferente no gráfico de resposta, de forma que no espectro resultante (callouts) informações sobre o tipo de produto podem ser avaliadas em tempo real. Além do LIF, foram utilizadas como técnicas complementares de perfilagem de Condutividade Elétrica (CE) e de cor de solo, utilizando a ferramenta SCOST. Para essa ferramenta são usados os mesmos equipamentos do UVOST (hastes, cabeamento, equipamento de penetração no solo etc.). Nesse caso, é utilizado o espectro visível da luz e a resposta é comparada com a escala Munsell de cor de solo. Esse método é particularmente útil para individualizar e classificar os horizontes do solo através da identificação visual de descontinuidades.

No caso da Condutividade Elétrica, uma corrente é enviada através do solo por dois contatos instalados na sonda. Esta corrente é medida juntamente com a tensão resultante em mili-Siemens por metro $(\mathrm{mS} / \mathrm{m})$. Em geral a condutividade é função da granulometria do material, do grau de umidade do meio e de características de eventuais contaminantes. Assim, solos argilosos ou mais úmidos apresentam maior condutividade; inversamente, solos arenosos ou com contaminação por produtos oleosos apresentam condutividade mais baixa. Dessa forma, a CE fornece dados sobre a estratigrafia e possíveis horizontes contaminados por hidrocarbonetos. 
Figura 6 - Curvas espectrais (Callouts) - Intervalos de comprimentos de onda captados pelo UVOST: $350 \pm 20 \mathrm{~nm}$ (querosene de aviação e gasolina); $400 \pm 20 \mathrm{~nm}$ (diesel); $450 \pm 20 \mathrm{~nm}$ (creosoto) e $500 \pm 20 \mathrm{~nm}$ (hidrocarbonetos não especificados) (MARTIN e ST GERMAN, 2008)

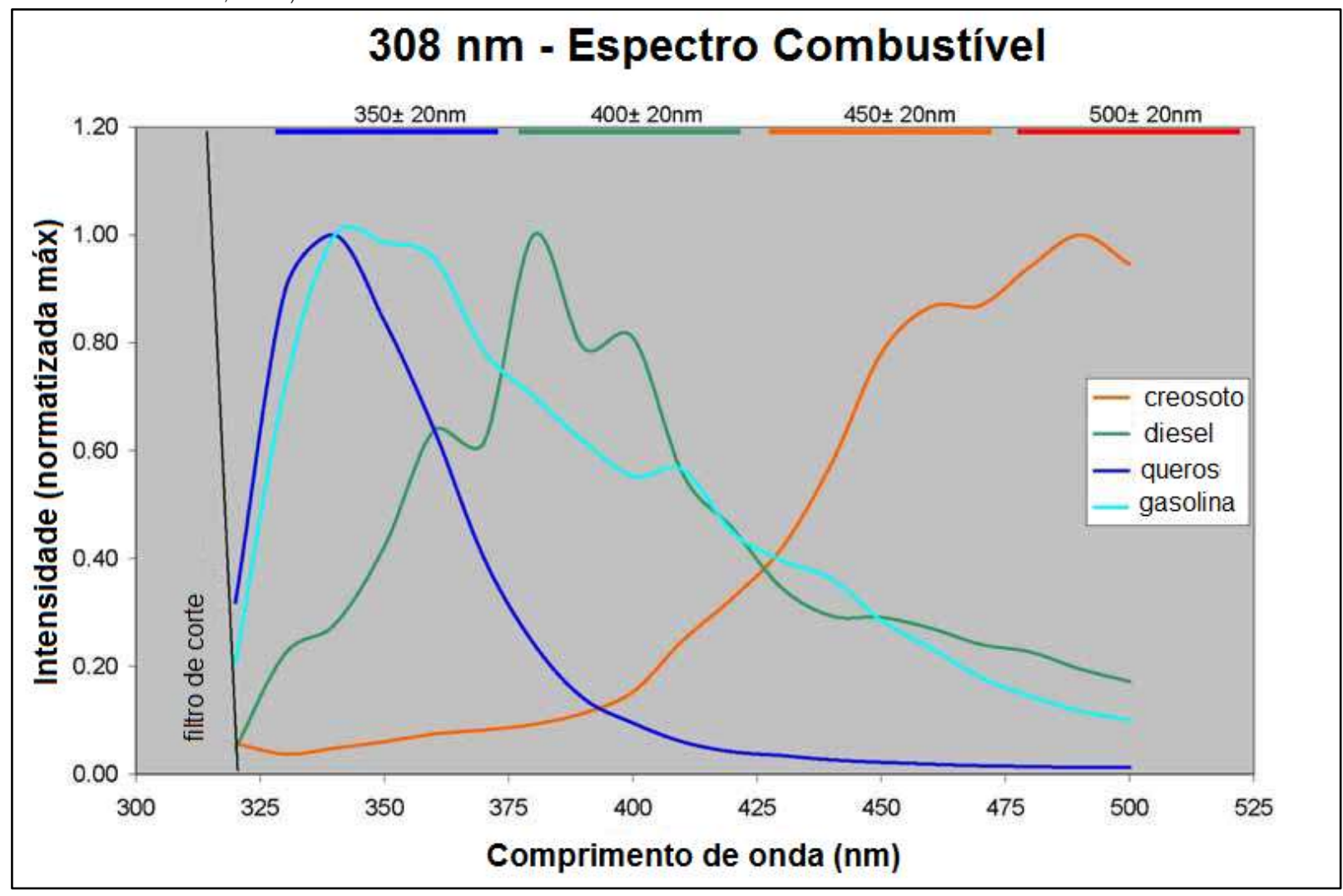

\section{RESULTADOS E DISCUSSÃO}

Diagnósticos ambientais previamente realizados na área indicaram a presença de material oleoso, na forma de lentes dispersas em solo orgânico, com extensão lateral e vertical variando de centimétrica a métrica. A heterogeneidade na distribuição foi atribuída á remobilização e movimentação do solo por máquinas durante o aterramento da área.

De forma geral, os resíduos apresentam menores concentrações de hidrocarbonetos mais leves. Análises de Hidrocarbonetos Totais de Petróleo (TPH) fracionados no resíduo, realizados em diagnósticos anteriores (GEORADAR, 2006), suportam essa afirmação e indicam predominância de concentrações de compostos aromáticos nas faixas de cadeia mais longa (C10 a C12 e C16 a C21), menos voláteis e mais persistentes no meio ambiente. Os compostos alifáticos apresentaram o mesmo padrão, fato que também foi atribuído ao elevado estágio de biodegradação no qual o resíduo se encontra (GEORADAR, 2006). Os ensaios de produto lixiviado e solubilizado realizados nos resíduos para caracterização conforme normas NBR não indicaram concentrações significativas de compostos orgânicos (GEOKLOCK, 2011).

A predominância de hidrocarbonetos mais pesados difere do que é esperado em contaminações recentes, principalmente por gasolina ou diesel, que apresentariam concentrações maiores de frações mais leves. Essas frações mais leves, quando submetidas à radiação ultravioleta apresentam fluorescência em comprimentos de onda na faixa aproximada de $330 \mathrm{~nm}$ a $420 \mathrm{~nm}$, enquanto que os resíduos oleosos de cadeias mais longas apresentam respostas em torno de $500 \mathrm{~nm}$.
Nos pontos avaliados nesse estudo as sondagens convencionais apontaram a presença de borra oleosa ou de produto oleoso impregnado no solo. As análises químicas indicaram em apenas dois pontos, sondagens 324 e 326, concentrações mesuráveis de TPH fracionado, principalmente alifático (21-32) de 8,89 e 8,3 $\mathrm{mg} / \mathrm{kg}$ respectivamente. Não foram detectados outros compostos orgânicos.

Tabela 1 - Correspondência entre os pontos de perfilagem UVOST, sondagem e perfil de cor do solo (soil color)

\begin{tabular}{cccc}
\hline Ponto & UVOST & Sondagem & Soil color \\
\hline 01 & LF1 & 326 & Scost01 \\
02 & LF2 & 325 & Scost02 \\
03 & LF3 & 324 & Scost03 \\
04 & LF4 & 469 & Não realizado \\
05 & LF5 & 577 & Não realizado \\
\hline
\end{tabular}

LIF 1

O local da perfilagem LIF1, junto à sondagem 326 (Figura 6) apresenta, em suas camadas mais rasas, a ocorrência de aterro em tons de marrom e bege, que em profundidade passa para tons de cinza e novamente bege a aproximadamente $6 \mathrm{~m}$ de profundidade, onde provavelmente atinge a zona de alteração da rocha subjacente (substrato). Odor característico e produto oleoso impregnado ao solo foram relatados no intervalo de 2,10 $\mathrm{m}$ a 2,40 $\mathrm{m}$ de profundidade. Foram coletadas duas amostras de solo para análises químicas. A primeira no intervalo de 3,80 a 4,20 m de profundidade e outra de 6,40 a $6,80 \mathrm{~m}$ no final da sondagem. 
Concentração de $8,30 \mathrm{mg} / \mathrm{kg}$ de TPH fracionado foi encontrado apenas na amostra coletada no primeiro intervalo de $3,80 \mathrm{~m}$ a 4,20 m; no outro nada foi detectado. Esta sondagem apresentou ainda um trecho de aproximadamente $1 \mathrm{~m}$, a partir de 2,40 m, sem recuperação e, consequentemente, sem informações sobre o limite inferior de ocorrência do produto oleoso.

Os resultados da perfilagem LIF 1 (Figura 7) mostram picos de resposta entre 1,80 m e 2,20 m de profundidade, indicando forte correlação com as profundidades onde o material oleoso foi descrito. Os logs de intensidade de fluorescência foram maiores para comprimentos de onda superiores a $450 \mathrm{~nm}$, o que é compativel com o tipo de contaminação descrita: material oleoso antigo, com predominância de frações mais pesadas de hidrocarbonetos.

Pelo perfil SCOST (Figura 7) foi possível verificar variações nas colorações de marrom para cinza e para bege com a profundidade. Embora não fosse efetivo para a identificação pontual do resíduo, foi eficiente para identificação da passagem do material de aterro para a zona de alteração do substrato rochoso.

O perfil de Condutividade Elétrica apontou variações em função da granulometria do material (arenoso/argiloso) e da presença de material oleoso. Embora com pouca precisão para detalhar essas variações, foi eficiente na delimitação do contato aterro/rocha subjacente.

Figura 7 - Ponto LF 1. Perfilagem LIF com CE e perfilagem cor do solo e CE

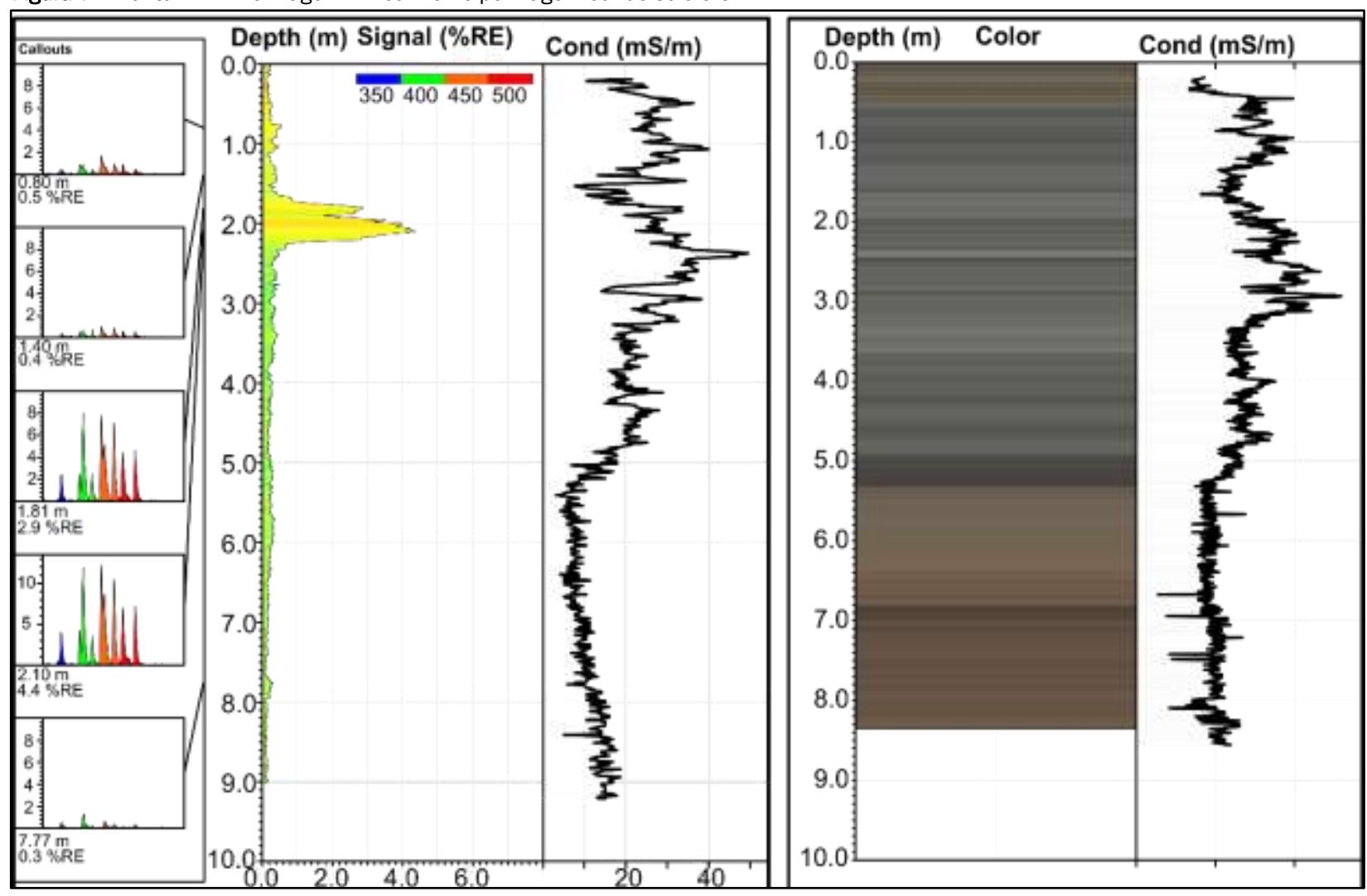

A Figura 8 apresenta uma comparação entre os métodos. Da comparação entre essas é possível verificar que pela perfilagem UVOST o material oleoso no solo ocorre na profundidade de 2,0 $\mathrm{m}$ e se correlaciona com o intervalo de menor CE, fato confirmado pela descrição do perfil de sondagem.

\section{LIF 2}

A perfilagem LIF 2 indica ocorrência de dois níveis com fase residual oleosa: um pico na profundidade de aproximadamente 2,80 $\mathrm{m}$ indica uma ocorrência com poucos centímetros de espessura; ocorrência mais espessa aparece no intervalo de 4,00 m a 4,50 $\mathrm{m}$ de profundidade. No caso deste registro mais espesso, verifica-se também uma diminuição expressiva na condutividade elétrica.

O perfil SCOST não permitiu discriminar o material oleoso da matriz do solo. Na porção superficial predomina o aterro de cores em tons de marrom, que passa abaixo para solo orgânico cinza até atingir a zona alteração do substrato rochoso, caracterizado pela cor bege. Novamente a transição para o saprólito é marcada por queda nos valores de condutividade elétrica.

Notar que, embora a perfilagem LIF 2 tenha sido realizada próximo à sondagem 325, a distribuição do resíduo ocorre em profundidades diferentes, refletindo a heterogeneidade na disposição do material. A sondagem 325 apresenta descrição litológica semelhante à da sondagem 326, passando de aterro de cor marrom amarelado a solo cinza claro/escuro e finalmente bege quando atinge o perfil de alteração da rocha subjacente. Foi descrita borra oleosa preta, com forte odor característico, no intervalo de 5,30 $\mathrm{m}$ a 5,40 $\mathrm{m}$ de profundidade; alteração da rocha subjacente (saprólito) foi descrita a aproximadamente 7,80 m (Figura 9). 
Figura 8 - Comparativo entre as técnicas de perfilagem LIF 1, cor do solo e CE, tendo como referência a sondagem 326

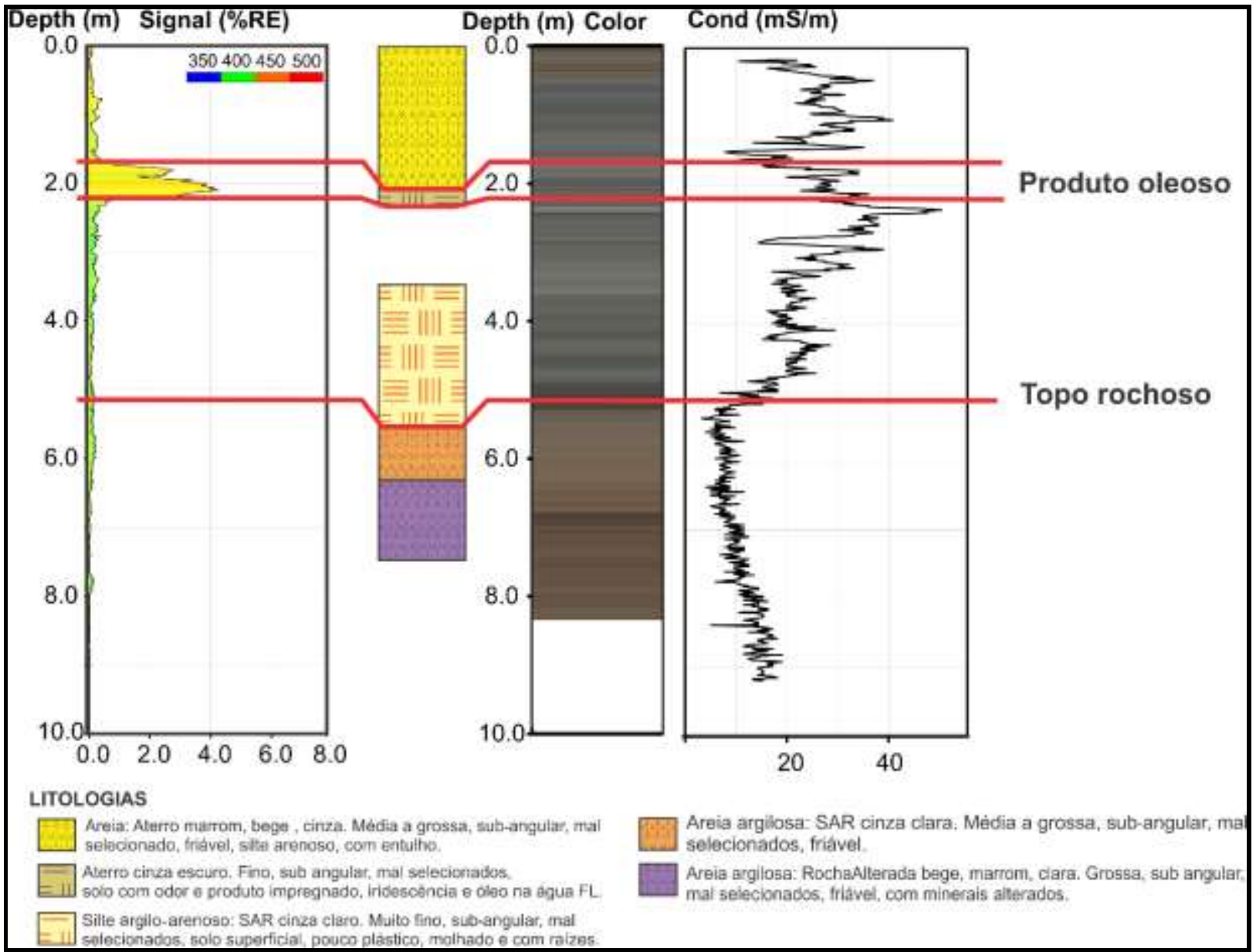

Figura 9 - Comparativo entre as técnicas de perfilagem LIF 2, cor do solo e CE, tendo como referência a sondagem 325

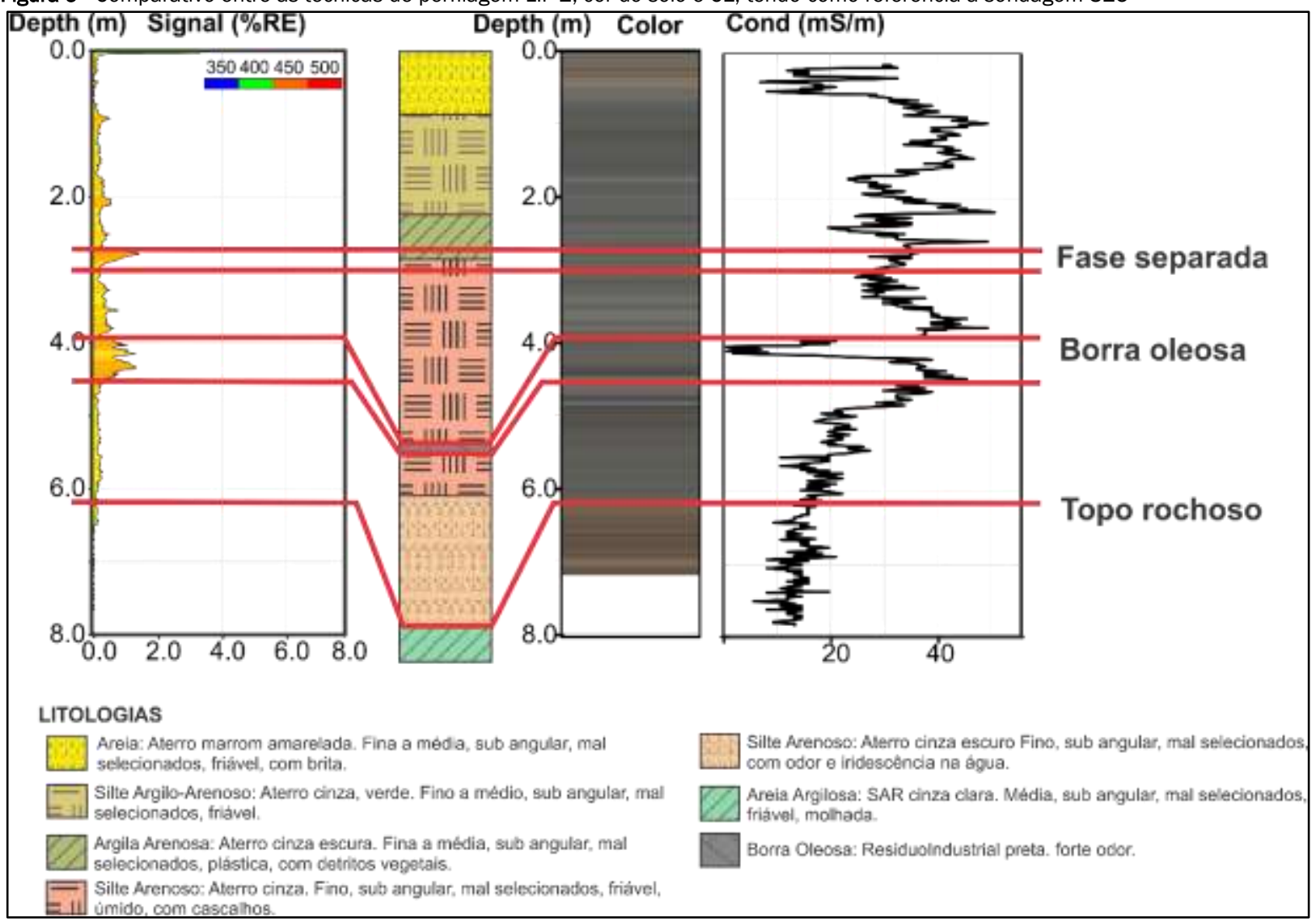


de alteração de rocha. Observa-se também a queda no CE no in-

A perfilagem LIF 3 identificou dois níveis com resíduos oleosos. 0 primeiro, a 0,80 $\mathrm{m}$ de profundidade, apresenta forte intensidade de resposta no comprimento de onda próximo a $400 \mathrm{~nm}$, característica de óleo diesel. Essa ocorrência foi atribuída a contaminação recente, provavelmente resultado de vazamento em equipamentos que trabalharam no local. 0 outro nível, no intervalo de 3,00 m a 4,40 m, apresenta vários picos de menor intensidade, sugerindo a presença de material oleoso disperso.

O perfil SCOST seguiu o mesmo padrão dos anteriores até a zona tervalo contaminado detectado pelo LIF 3.

Comparando-se os dados das diversas fontes (Figura 10) verificase que a sondagem 324 mantém o padrão das anteriores, com aterro bege mais superficial, seguido abaixo por solo orgânico cinza até a zona de alteração do substrato rochoso, em torno de $7 \mathrm{~m}$ de profundidade, em cor bege e caracterizado pela presença de minerais alterados. Nesse perfil não foi descrito material oleoso ou odor característico, mas foi detectado TPH alifático $(8,89$ $\mathrm{mg} / \mathrm{kg}$ ) em amostras coletadas no intervalo de 5,20 m a 5,60 m de profundidade.

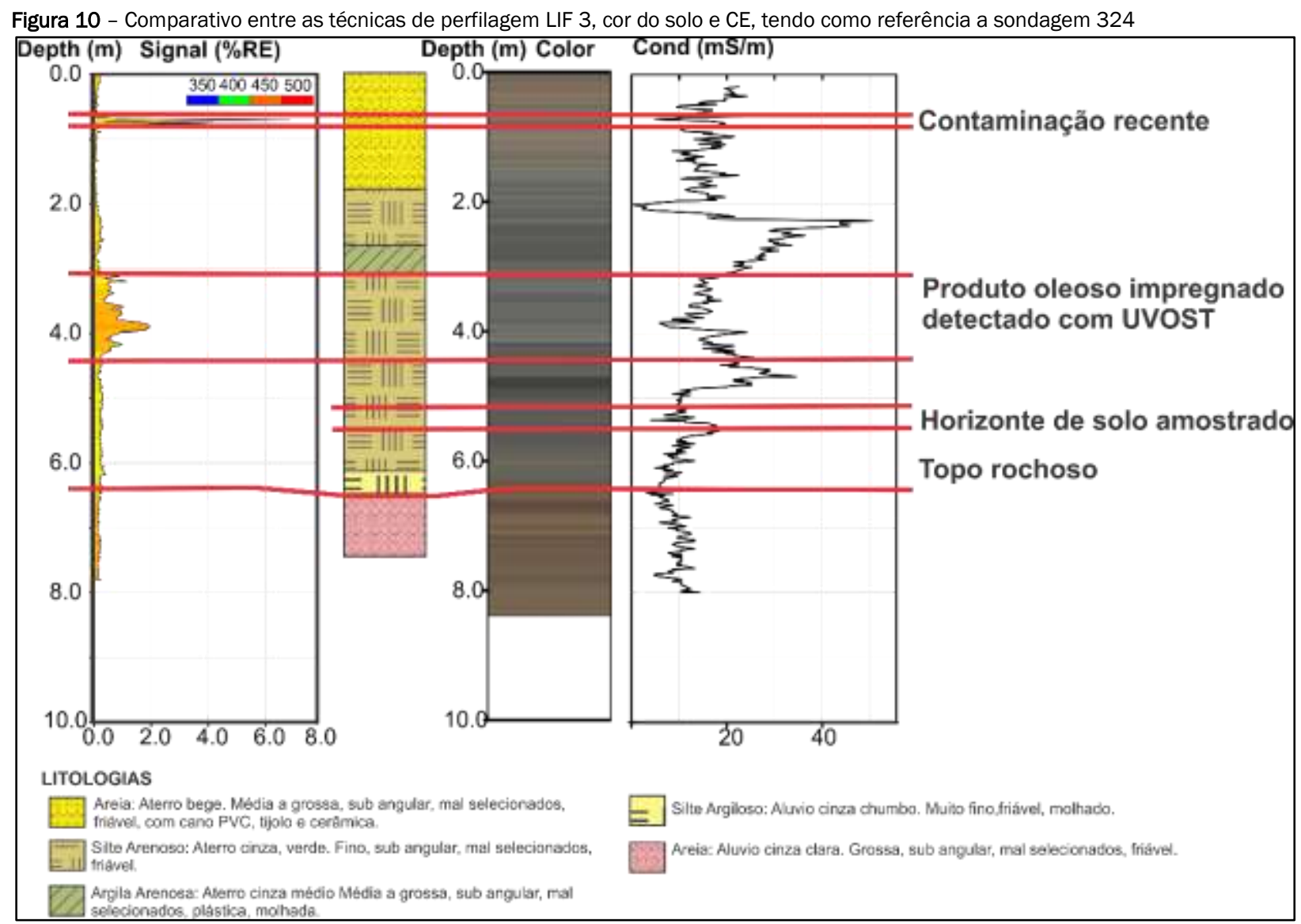

LIF 4

A perfilagem LIF 4 apontou presença de material oleoso entre 2,50 m e 3,40 m, com predominância de resposta em comprimentos de onda na faixa de $500 \mathrm{~nm}$, característico de produtos mais pesados. Estes dados são fortemente correlacionáveis com as descrições anteriores. O intervalo contaminado mostra a mesma diminuição da CE.

Neste ponto não foi realizada perfilagem SCOST, e a sondagem não atingiu a zona de alteração da rocha subjacente. A sondagem 469 mostra, em superfície, aterro de cor marrom a bege que passa em profundidade para solo orgânico cinza. Foi detectado produto oleoso impregnado no solo com forte odor característico, no intervalo de 3,00 m a 4,00 $\mathrm{m}$ de profundidade, e presença de óleo na água a 2,50 m (Figura 11).
LIF 5

As sondagens e perfilagens desse ponto foram realizadas em via pública. O perfil litológico da sondagem 577 é semelhante aos anteriores, com produto impregnado, odor e óleo na água no intervalo de 2,10 $\mathrm{m}$ a 3,00 $\mathrm{m}$ de profundidade. 0 intervalo amostrado para análises químicas situou-se logo abaixo do horizonte com material oleoso, não sendo encontradas concentrações mensuráveis de hidrocarbonetos.

Foram detectadas duas respostas distintas de fluorescência associadas à presença de hidrocarbonetos. Uma, superficial, até aproximadamente $0,40 \mathrm{~m}$, com predominância de comprimentos de onda na faixa de $400 \mathrm{~nm}$ a $450 \mathrm{~nm}$, envolvendo diesel e produtos mais pesados, provavelmente relacionados a vazamentos recentes, hipótese esta reforçada pelo fato de esta sondagem ter sido realizada em via pública. Outra, entre 1,60 m e 2,80 m de 
profundidade, com resposta de maior intensidade e comprimentos de onda na faixa de $450 \mathrm{~nm}$ a $500 \mathrm{~nm}$, característicos de produtos mais pesados, o que é compatível com produtos anti- gos descritos na sondagem. Este horizonte apresenta forte correlação com a descrição da sondagem que indicou produto impregnado (Figura 12).

Figura 11 - Comparativo entre as técnicas de perfilagem LIF 4 e CE, tendo como referência a sondagem 469

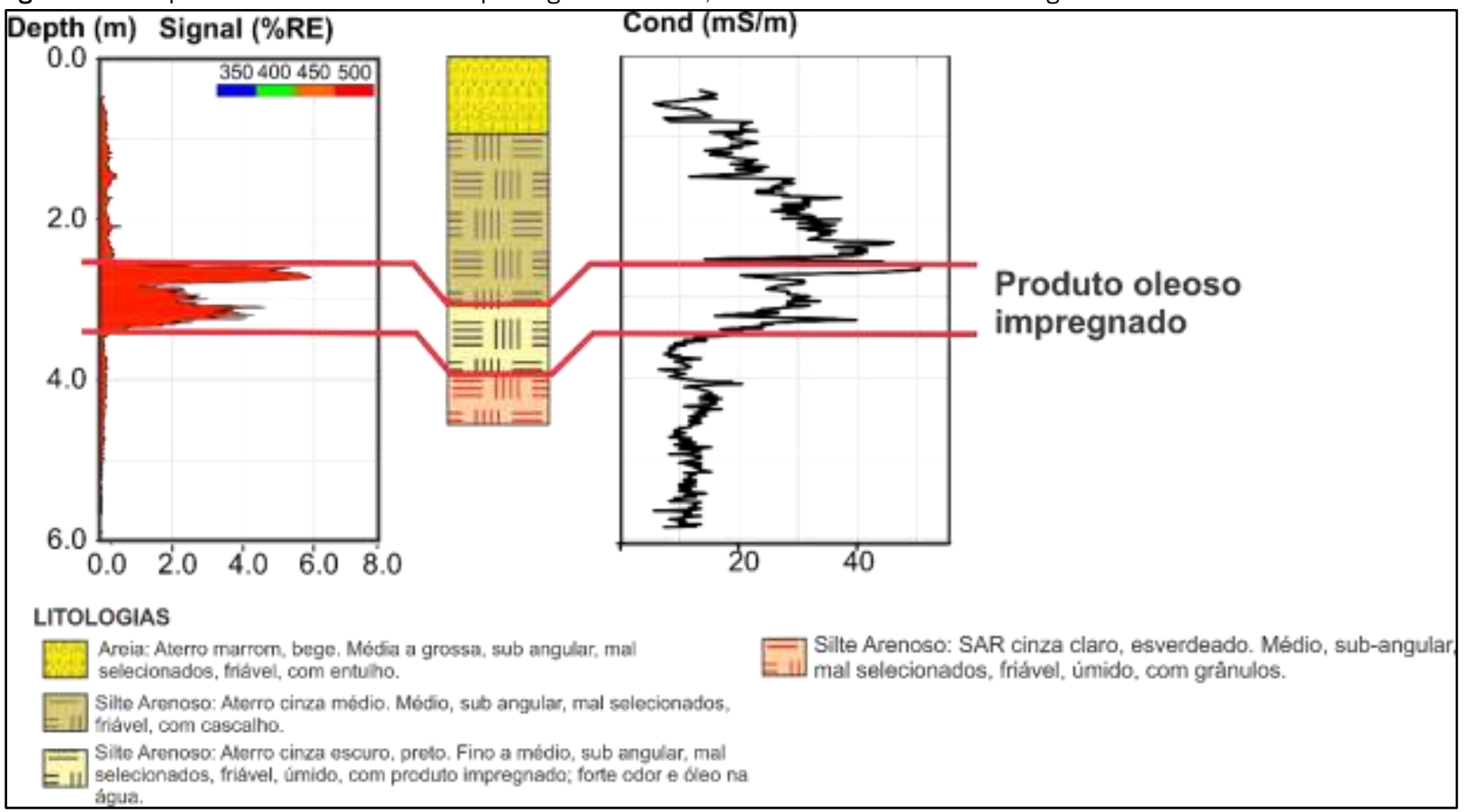

Figura 12 - Comparativo entre as técnicas de perfilagem LIF 5 e CE, tendo como referência a sondagem 577

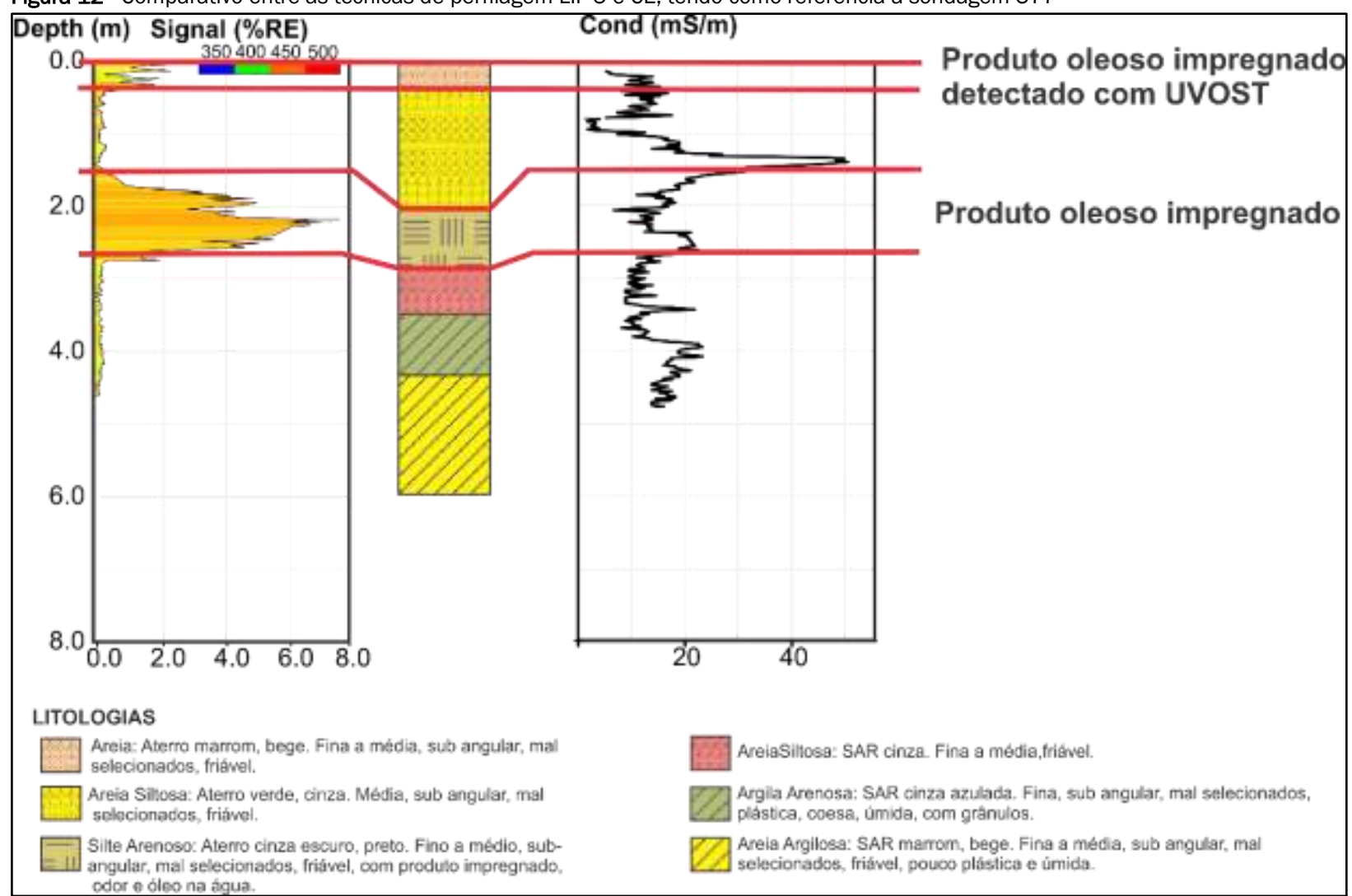


Da mesma forma que nos pontos anteriores, o intervalo contaminado com material oleoso coincide com a queda observada no perfil de CE.

O LIF representa uma ferramenta eficiente para identificação semiquantitativa de hidrocarbonetos na fase móvel, trapeado ou residual em sub-superfície, como demonstrado por diversos trabalhos, incluindo Löhmannsröben \& Roch (2000), Lamb (2012), Isler et al. (2013), Suthersan et al. (2016). As vantagens do emprego LIF incluem menor tempo de investigação, aquisição de informações de modo contínuo no intervalo de profundidade investigado e possibilitar a modificação da malha de amostragem em razão dos resultados obtidos em tempo real durante a investigação, possibilitando um diagnóstico ambiental mais consistente e conclusivo.

Por outro lado, os métodos tradicionais de investigação para detecção de compostos orgânicos no solo se baseiam em coletas pontuais de amostras de solo para determinação da concentração de hidrocarbonetos. Estas técnicas estão sujeitas à imprecisões, uma vez que os pontos de amostragem previamente determinados podem não ser representativos e a densidade de amostras por ponto pode ser inadequada para caracterização do problema investigado. Cabe ressaltar, ainda, a existência de problemas operacionais como a não-recuperação de solo em intervalos específicos e compressão do solo no amostrador.

A intensidade da fluorescência pode ser correlacionada com a quantidade de hidrocarbonetos poliaromáticos (HPAs) no solo (LÖHMANNSRÖBEN \& ROCH, 2000). Entretanto, em razão da não identificação de HPAs no solo, não foi possível correlacionar quantitativamente os resultados do LIF com as concentrações de HPAs no solo. Entretanto, os valores mais elevados de \%RE foram compatíveis com os intervalos de profundidades onde se constatou que a borra oleosa foi depositada.

Nesses cinco exemplos apresentados verificou-se que não é possível determinar a exata posição da zona contaminada utilizandose somente testemunhos de sondagens (liners). Invariavelmente, todas as porções contaminadas identificadas nos testemunhos estão deslocadas e, em muitas situações, os intervalos descritos são menores (Figuras 8, 9, 12) ou mesmo ausentes (Figura 10). Operacionalmente, na perfuração, é muito difícil obter recuperação de $100 \%$ de material, visto que sempre ocorre perda de material e/ou compactação dentro do tubo coletor (Iiner). Adicione-se a isso a deformação causada pelo transporte do material.

A cor do substrato, que resulta da combinação de todos os constituintes presentes, não permitiu identificar as zonas contaminadas. Já nos perfis de CE observou-se diminuição da condutividade na zona contaminada identificada pelo UVOST. No entanto, como a CE é uma resposta composta por uma combinação da matriz sólida com o fluido intersticial, esta correspondência tem que ser vista com cautela.

\section{CONCLUSÕES}

A aplicação do método LIF em campo, utilizando UVOST, se mostrou eficiente na detecção de resíduos oleosos e de material oleoso impregnado no solo, independente do longo tempo de depo- sição dos resíduos, de seu grau de intemperismo e da heterogeneidade na distribuição espacial da contaminação.

A forma e a composição do sinal de resposta de fluorescência foram coerentes com o tipo de material encontrado, em que predominam hidrocarbonetos de cadeias maiores. A técnica também foi sensível a lentes pouco espessas de resíduos de hidrocarbonetos e na indicação da presença desses produtos na zona saturada, mesmo em pequena quantidade (película).

O perfil de cor do solo, em função da ocorrência de argila orgânica escura e de aspecto visual semelhante ao resíduo oleoso, não se mostrou eficiente para a delimitação das lentes de contaminantes, sendo mais precisa na delimitação do saprólito, dado que a variação na cor foi significativa.

A Condutividade Elétrica, embora localmente apresentasse alguma resposta, também não se mostrou um bom parâmetro para identificação do material oleoso, porém apresentou boa indicação na transição do aterro para a zona de alteração do substrato rochoso.

A precisão obtida com o UVOST/LIF indica que este constitui uma importante ferramenta para delimitação tridimensional de resíduos oleosos, indicando em tempo real e com menores investimentos a área passível de remediação. Além disso, permitiu a identificação e semiquantificação de contaminantes presentes, o que favorece a tomada de decisões em campo e possibilita o direcionamento da amostragem para áreas realmente contaminadas, tornando a investigação mais rápida e reduzindo os custos.

Este estudo permitiu verificar que as técnicas tradicionais de investigação apresentam uma tendência de indicar espessuras menores de solo contaminado, gerando cálculos imprecisos do volume de solo contaminado, subestimando o volume de solo a ser remediado.

\section{REFERÊNCIAS}

BRAVO, S.R. Fluorescence. environmental applications encyclopedia of analytical science. Second Edition. Elsevier. p 178-186, 2005.

BUJEWSKI, G.; RUTHERFORD, B. The rapid optical screening tool (rost) laser-induced fluorescence (lif) system for screening of petroleum hydrocarbons in subsurface soils. Innovative Technology Verification Report. 86 p. 1997. http://www.epa.gov/etv/pubs/01_vr_fugro.pdf. Acessado em 24/03/2015.

CRUMBLING D. M. Summary of Triad Approach. U.S. Environmental Protection Agency Office of Superfund Remediation and Technology Innovation: Superfund Triad Support Team. 2004. http://www.triadcentral.org/ref/doc/ triadsummary.pdf acessado em 22/11/16.

FUNDUNESP - Fundação para o Desenvolvimento da UNESP. Projeto básico de remediação área do Bairro Itatinga. 60 p. 2011

FUNDUNESP - Fundação para o Desenvolvimento da UNESP. Relatório de avaliação técnica: avaliação de resultados analíticos em ponto de surgência localizado à Rua Tancredo Neves - Bairro Itatinga. 186 p. 2013.

GEOKLOCK. Diagnóstico ambiental detalhado: fase III: área azul - Itatinga - São Sebastião, SP. 3.935 p. 2011. 
GEORADAR. Relatório de diagnóstico ambiental.: bairro Itatinga: área vermelha - São Sebastião, SP. 68 p. 2006.

GEORADAR. Relatório de diagnóstico ambiental: bairro Itatinga: área azul - São Sebastião, SP. 61 p. 2007 a.

GEORADAR. Adendo ao relatório de diagnóstico ambiental: bairro Itatinga: área azul- São Sebastião, SP. 18 p. 2007b.

HADLEY, P. W.; NEWELL, C. The New Potential for Understanding Groundwater Contaminant Transport. Groundwater, v. 52, n. 2. P. 174-186. 2014.

HESS, E.; HAMILTON, D. Rapid Optical Screen Tool (ROSTTM): Innovative Technology Evaluation Report. USEPA Office of Research and Development, Washington, DC, 69 p. 1995.

ISLER, E.; ET al. Uso do Laser-Induced Fluorecence (LIF) na caracterização de áreas contaminadas em tempo real. Anais do III Congresso Internacional de Meio Ambiente Subterrâneo. p. 2179-9784. http://aguassubterraneas.abas.org/ asubterraneas/issue/ view/1298. Acessado em 26/03/2014. 2013.

ITRC - THE INTERSTATE TECHNOLOGY \& REGULATORY COUNCIL. DNAP:. Guidance document integrated dnapl site characterization and tools selection. 361 p. 2015.

KARLITSCHEK, P. et al. Detection of aromatic pollutants in the environment by using UV-laser-induced fluorescence. Applied Physics B. v. 67, n. 4, p. 497-504, 1998.

LAMB, S. R. Sampling and Analysis Plan LNAPL Laser Induced Fluorescence / Membrane Interface Probe Investigation. Troy Mills Landfill Superfund Site Troy, New Hampshire. 252 p. 2012.

LÖHMANNSRÖBEN, H.-G.; ROCH, Th. In situ laser-induced fluorescence (LIF) analysis of petroleum product-contaminated soil samples. Journal of
Environmental Monitoring, v. 2, n. 1, p. 17-22, 2000. Doi: 10.1039/A906638A

MONDELLI, G. et al. Investigação geoambiental aplicada a planos de intervenção em guia de elaboração de planos de intervenção para o gerenciamento de áreas contaminadas. Capítulo 2. 1. ed.rev. IPT, 2014. 398 p.

MURPHY, B. L.; MORRISON, R.D. Introduction to environmental forensics. Academic Press, 704 p. 2014.

RIYIS, M. T. Investigação geoambiental com tomada de decisão em campo utilizando o piezocone de resistividade como ferramenta de alta resolução. Dissertação de Mestrado em Engenharia Civil e Ambiental Faculdade de Engenharia de Bauru, Universidade Estadual Paulista “Júlio de Mesquita Filho". 149 p. 2012.

SANTOS, A. D. et al. Aprimoramento do modelo conceitual da área através do uso de ferramentas de investigação de alta resolução. InterfacEHS Saúde, Meio Ambiente e Sustentabilidade. v. 10, n. 2. p. 03-22. 2015.

SUTHERSAN, Suthan S. et al. Rethinking conceptual site models in groundwater remediation. Groundwater Monitoring \& Remediation, v. 36, n. 4, p. 22-30, 2016. Doi: 10.1111/gwmr.12192

USEPA - UNITED STATES ENVIRONEMENTAL PROTECTION AGENCY. EXpedited site assessment tools for underground storage tank sites: a guide for regulators. Office of Underground Storage Tanks, OSWER. USEPA 510B-97-001. 5 p. 1997.

USEPA - UNITED STATES ENVIRONEMENTAL PROTECTION AGENCY. Record of decision: soil and NAPL operable unit del amo facility superfund site, Los Angeles. CA, 2011. $210 p$

USEPA - UNITED STATES ENVIRONEMENTAL PROTECTION AGENCY. High resolution site characterization: contaminated site clean-up information (Clu-In). https://clu-in.org/characterization/technologies/hrsc/hrscintro.cfm. Acessado em 21/11/16. 2013. 